\section{R.E. property meets technology: cross-country comparison and general framework}

\author{
Chiara Tagliaro, Stefano Bellintani and Gianandrea Ciaramella \\ Department of Architecture, Built Environment and Construction Engineering, \\ Politecnico di Milano, Milan, Italy
}

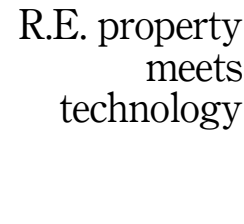

125

Received 27 September 2019 Revised 27 February 2020 16 March 2020

Accepted 17 March 2020

\begin{abstract}
Purpose - Due to the young age of proptech, little is known about the dynamics of its expansion. In particular, there is limited agreement about a definition of "proptech," while different categorizations are popping up. A severe lack of information emerges for the proptech scenario in Italy. The goal of this paper is to systematize multiple proptech maps in the attempt to create a framework for comparison of country-specific trends and an overarching definition of proptech. The research examines the evolutionary stage of the Italian digital real estate sector and compares it to the international context.

Design/methodology/approach - An in-depth analysis of 12 proptech maps at both national and international level was conducted based on online research. A list of Italian proptech companies was composed through multiple methods. A map was built for a cross-country comparison.

Findings - Each country or organization tends to develop its own categorization. This creates a multifaceted context where comparison and analysis are challenging. The Italian proptech sector seems underdeveloped compared to neighboring countries. Big room for improving the proptech business in this country still exists. Practical implications - The results are valuable for proptech start-ups, business investors and wellestablished real estate actors to build on new entrepreneurial initiatives. The opportunity to advance proptech mapping and categorization emerges as a prospect for future research.
\end{abstract}

Originality/value - This research adds an overview of cross-country proptech categories and proposes the first analysis of Italian proptech. This will contribute to support entrepreneurial opportunities.

Keywords Proptech, Italy, Real estate, Digitalization, Clusters, Categories

Paper type Research paper

\section{Introduction}

Proptech is a recent phenomenon, but it has been attracting increased attention from several players.

According to data published by Venture Scanner in June 2019 (https://www. venturescanner.com/), the proptech market has undergone a sudden acceleration in terms of funding. 2019 is the year in which the sector recorded the largest amount of investment (9.3bn dollars through the first and second quarters). This amount represents $50 \%$ of the total

(C) Chiara Tagliaro, Stefano Bellintani and Gianandrea Ciaramella. Published by Emerald Publishing Limited. This article is published under the Creative Commons Attribution (CC BY 4.0) licence. Anyone may reproduce, distribute, translate and create derivative works of this article (for both commercial and non-commercial purposes), subject to full attribution to the original publication and authors. The full terms of this licence may be seen at http://creativecommons.org/licences/by/4.0/legalcode

This paper forms part of a special section "PropTech and Entrepreneurship - Innovation in Real Estate II", guest edited by Dr Larry Wofford, Dr David Wyman, Dr Elaine Worzala.

This paper originated from the valuable work of two students of Politecnico di Milano who successfully ended their master's program in Management of Built Environment with two thesis on the topic of proptech. Merilisa Spaltro with a thesis entitled "Evolution of Italian real estate: a proposal for digital real estate observatory" (https://www.politesi.polimi.it/handle/10589/143463) and Marco Magnani with a thesis entitled "Main trends in the international Proptech market: proposal for a real estate tokenisation start-up" (https:/www.politesi.polimi.it/handle/10589/143028). The authors would like to thank them for their great contribution to our elaborations.

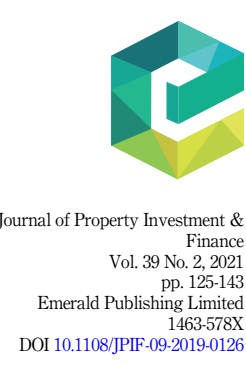


JPIF

39,2

funding in 2018 and $140 \%$ of the funding up to the second quarter of 2018 . Also, the amount of investment capital collected in the late stages of funding has been increasing significantly since 2014 (Venture Scanner, 2018a), which proves that the global market is consolidating and rapidly reaching a more mature stage.

In the global panorama, the United States leads the investments in this industry, both in the number of funding rounds and in the amount of funding raised (Singh, 2018). The United Kingdom and China follow with investments that are proportional to the dimension of each country. Other European countries demonstrate that they play a role on a global scale, such as Spain, France, The Netherlands, Germany, Finland and Poland.

Not only are investors involved in this evolution, but also developers, occupiers, employees and retailers among other real estate players (Stanley, 2018). Several proptech events are bringing all these relevant stakeholders together. Just to name a few: MIPIM (http://www.mipim.com), the largest global real estate event (held every March in Cannes, France), recently introduced a forum dedicated to the proptech business (https://www.mipimproptech.com/en.html) that has been taken worldwide over the past year, including New York in early November, Hong Kong in late November and Paris scheduled for June-July 2020; FUTURE Proptech (https:/futureproptech.co.uk/) turned five years old in 2019 as a global debate and exhibit platform; Propteq Europe (https:/propteqclub.com/proptech-europe2019/) reached its fourth event with the Global Summit in September 2019, held in London, where around 300 organizations from around the world participated in; and even the Royal Institution of Chartered Surveyors (RICS) held its first Proptech Conference on November 23, 2018 (https://www.rics.org/en-hk/events).

Besides, technology incubators have been established in universities all over the world, including DMZ at Ryerson University (Toronto, Canada); New Venture Institute at Flinders University (Adelaide, Australia); Tsinghua x-lab at Tsinghua University (Beijing, China); PoliHub at Polytechnic University of Milan (Milan, Italy); StartX at Stanford University (Stanford, CA.); and more (Stanley, 2018).

These events and initiatives promote the potential development of the sector and informal conversations.

However, a systematic analysis of the sector is missing, while an agreed definition of proptech is still to come. Moreover, the experience of different countries in this field remains understudied.

\subsection{Definitions of proptech}

The term "proptech" has become a buzzword. Despite the relative frequency of Web searches based on the word "proptech" and encouraging market trends, the definition of this term is fuzzy. It derives from the crasis of "property" and "technology." It includes all products, processes and business ideas that apply the most innovative resources in the information communication technology (ICT) realm (Hasenmaile and Rieder, 2017). Expressions such as "CRETech" (Putzier, 2016), "Real EsTech" (Pyle et al., 2017), "Real Estate Tech Companies" (CBInsights, 2017), but also "RealTech" are often used as synonyms for similar types of business. "FinTech" and "ConTech" refer to technological applications in the financial and construction sectors, but are often difficult to distinguish from proptech (Maududy and Gamal, 2019a).

Commercial players, including KPMG (Pyle et al., 2017, p. 5) and RICS (2018), agree on defining "proptech" as a general term referring to all the aspects that cover how technology and digital innovations affect the built environment.

James Dearsley, guru of the UK proptech sector and founder of Unissu (https://www. unissu.com/), in collaboration with Professor Andrew Baum from the University of Oxford, elaborated a definition of proptech entailing a more complex concept. According to them, proptech is, on the one hand, a name defining all the technological innovations in the property segment and, on the other hand, the industry itself, the business sector and, more generally, 
"a movement driving a mentality change with the real estate industry" (Dearsley, 2018). Companies that bring about this movement are also called proptech. These are mainly startups (Hasenmaile and Rieder, 2017; Maududy and Gamal, 2019a) even though many smallmedium enterprises (SMEs), corporations and unicorns have been playing a significant role in the introduction of technology in the real estate sector (Baum, 2017).

Shaw (2018) argues that the term "proptech" does not explain sufficiently the sociotechnical dynamics driving the digitalization of real estate technology. He proposes the concept of "Platform Real Estate" to highlight the fact that digital infrastructures are enabling new network effects and interactions. A similar argument is raised by Porter $e t a l$. (2019) stating that new technologies are restructuring social and economic relationships in real estate.

What is common between all these definitions is that the main objective of proptech is to optimize the traditional value chains of real estate and to obtain more efficiency and effectiveness (Pyle et al., 2017; Maududy and Gamal, 2019a). The three main branches of real estate have been involved in this wave of change, namely facility, property and asset management. However, proptech companies also have to do with the real estate market, software and databases and the Internet of things (IoT). The proptech sector comprises a variety of business types that range from real estate management to financial services, transaction, construction, data exchange, maintenance and facility management services. While these loose boundaries explain the difficulty in elaborating a univocal and stable definition of proptech, several maps have been built to identify the main areas that are influenced by this nascent phenomenon.

\subsection{Approaches to taxonomy}

A review of the available white and grey literature reveals that most authors rely on two distinct approaches in the attempt to identify proptech areas, namely classification and categorization. According to Jacob (2004), classification happens on the basis of predetermined guidelines or principles and through a scheme that is artificial and arbitrary: "[...] artificial because it is a tool created for the express purpose of establishing a meaningful organization; and arbitrary because the criteria used to define classes in the scheme reflect a single perspective of the domain to the exclusion of all other perspectives." (Jacob, 2004, p. 522) While the approach to categorization is inductive, reasoning in support of classification is deductive. Categories are inferred from a creative synthetic process. Classes are derived from literature and are subsequently filled with entities.

Classification of proptech mainly stems from the following criteria:

(1) implemented technology, which can be distinguished in evolutionary stages such as Proptech 1.0, 2.0 and 3.0 (Baum, 2017; JLL, 2017);

(2) supply/value chain or development process, which is subdivided into subsequent stages: (1) preconstruction, (2) construction and (3) postconstruction (Maududy and Gamal, 2019b);

(3) drivers, such as information, transaction/marketplace and management/control (Baum, 2017) or production, construction and operation, management and marketing and transaction (Maududy and Gamal, 2019a);

(4) involved stakeholders, who can be clustered into four main market segments, namely capital investment activity, commercial market, building management and residential market (Shaw, 2018).

On the contrary, categorization is mainly based on types of activities or product function. This approach has been proposed mainly by consulting companies, but also researchers and

R.E. property meets technology 
JPIF

39,2

institutional sources apply it as an effective lens for market analysis. Categorization originates from technology and service mapping and allows for clustering the business in different sectors. To date, proptech mapping largely relies on snowball sampling based on participant observation and self-identification of existing companies (e.g. Baum, 2017; Shaw, 2018). This way, categorization attempts have led to various results.

The two approaches to classification and categorization happen to overlap in some cases (e.g. Baum, 2017; JLL, 2017). This encourages an overcoming of the local peculiarities of the market. However, an overarching principle suitable for reading the proptech phenomenon and trends still needs to be elaborated. Dissimilar interpretations of proptech subsectors are sprouting, often depending on country-specific dynamics. Some organizations regularly publish updated regional maps. However, some countries lag behind in the representation of local proptech trends.

\subsection{European context}

By August 2019, there were a total of 3,219 companies active in the proptech sector in Europe (Unissu, 2019). Among the various countries, some stand out for the number of companies. These are also the geographical contexts where the greatest number of events and debates are concentrated.

In the United Kingdom, Unissu counts about 805 proptech companies (https://www.unissu. com/proptech-resources/proptech-europe), mainly concentrated in London. For Germany, Gewerbe Quadrat (https://www.gewerbe-quadrat.de/proptech-markt/) records an increase in the number of companies from 229 to 238 from October 2018 to October 2019. However, with 2019 the count also includes 34 ConTech. In Spain, Spanish Estate (https://www.spanishestate. com/proptech), which draws on a Spanish website https:/www.hispacasas.com/proptech), identified 305 companies in May 2019 (compared to November 2018 when there were 281). Proptech Finland (http://proptechfinland.org/) reports a total of 136 proptech start-ups in Finland, 195 in Sweden, 90 in Norway and 120 in Denmark. Although the numbers do not stand out compared to other European countries, Finland is the country with the highest concentration of proptech (17 companies per million inhabitants) (Sipilä and Haataja, 2019). Credit Suisse (https:/www.credit-suisse.com/ch/en/articles/asset-management/swiss-proptech-report-2019-201905.html) mentioned over 200 swiss companies in 2019.

Initiatives to promote and enhance proptech businesses are also growing, bringing together local and national networks. Among these are Proptech Map (https://www. proptechmap.com/) and Proptech House (https://www.proptechhouse.eu/). The first network organizes the Builtworld Innovation Contest, through which it identified over 2,400 European proptech start-ups in 2019. The main represented countries are: Germany, the Netherlands, United Kingdom, Switzerland, Norway, Spain, Austria, France and Israel. Only one Italian start-up appears in this network. Proptech House, instead, was originally created by five national networks together with Workero. Currently this platform constitutes an alliance of $20+$ national hubs, but Italy does not appear in it.

Overall, the EU is composed of 28 national proptech markets, with different languages, skills, industry standards, processes, juridical frameworks (PropTech house, 2019). An attempt to consolidate this fragmented context should begin with (1) including missing countries in the current debate about proptech; (2) systematizing categories of proptech segments; and (3) building up a broadly applicable definition of proptech.

\section{Gaps and methodology}

Scientific debate about proptech is recent and academic contributions are scarce (Käki, 2018). When searching through Scopus for the word "proptech," only six scientific papers appeared as of February 2020. They have been published from 2018 on. The majority have a product- 
specific focus (e.g. blockchain and preventive maintenance), while only two face the topic from a general and theoretical point of view (Shaw, 2018; Maududy and Gamal, 2019b).

A common definition of "proptech" is missing, which makes it challenging to define investments and market segments that have been involved in this new digital wave. It is still unclear what technologies and actors are participating in the digitalization of the property sector and what their innovation potential is. Finally, some countries seem more active than others in keeping up with the pace of change.

This paper aims to:

(1) Analyze existing proptech categories with a bottom-up approach to derive a common framework for cross-country comparison;

(2) Advance the current knowledge on Italian proptech, which is still isolated from the international scene; and

(3) Propose a critical comment on extant definitions of proptech.

In order to reach the aforementioned goals, the research was developed through subsequent steps:

(1) Collection and analysis of several proptech maps, to outline recurring clusters;

(2) Focus on the Italian market, which is absent from the popular networks; and

(3) Elaboration on the match between the Italian market and other proptech maps, to propose a proptech framework and definition through inductive reasoning.

Web searches for "proptech map" were conducted in December 2018 and allowed collection of a number of diagrams and schemes that have been compared to one another. About 12 proptech maps were thoroughly analyzed, five of which at supranational level (i.e. CBInsight, MIPIM, VS, JLL, Proptech Map) and seven at national scale (i.e. United Kingdom, Germany, Spain, France, Finland, The Netherlands and Poland). By their cross-comparison, some transversal clusters were obtained.

Italian innovative ventures related to digital real estate were scouted in the same month through four main sources: Internet, Fintastico online platform, Assolombarda database and PoliHub incubator. In addition, personal contacts of the researchers and snowball sampling allowed an inclusion of some more companies in the analysis. For each company, several characteristics were analyzed including year of foundation, geographical localization and the description of the business that was used for subsequent categorization.

Google searches were performed on the Internet through the following combination of keywords: "proptech Italia," "startup innovative real estate Italia," "immobiliare innovazione" (eng. "real estate innovation" and "real estate Italy"). The most reliable websites were then selected and analyzed in depth. From this process only 14 proptech companies emerged, which could be properly attributable to the proptech sector.

Fintastico (https://www.fintastico.com/) is an online platform started in Italy around 2016 with the aim of giving users the opportunity to identify the digital services suitable for their needs, by selecting hundreds of fintech companies on the Web. Fintastico contains also a section that is specifically dedicated to proptech businesses. This section collects proptech companies that voluntarily promote their existence and want to appear on the platform, or Fintastico organization scouts for new companies itself through its contacts and expertise. From here, we gathered four proptech companies.

Assolombarda (http://www.assolombarda.it/english-version) is an Italian association of enterprises based in the Lombardy region. It currently expresses and protects the interests of about 6,000 companies of all sizes: small, medium and large, national and international,

R.E. property meets technology 
JPIF

39,2

130

producing goods and services in all product sectors. Assolombarda plays a fundamental role in terms of representation and lobbying. Assolombarda shared with us a list of its associated start-ups active in the following business areas: architecture, energy, design and furniture, innovative tertiary and chemistry and commerce. These number about 200, but only 13 of them could be identified as proptech start-ups.

PoliHub (http://www.polihub.it/en/) is the start-up incubator of Politecnico di Milano (Milan, Italy). It is the second biggest university incubator in Europe and the fifth in the world. PoliHub's mission is to support highly innovative start-ups with scalable business models and to push cross-fertilization processes between academia, start-ups and consolidated companies that are committed to innovation. On a dedicated webpage, they present all the start-ups that are joining the hub. However, only two of them could be recognized as proptech companies.

In the end, through word of mouth and snowball sampling, another ten companies were added to the list.

By reading the descriptions of the companies available on their respective websites, it was possible to aggregate them in different categories. These categories were compared to the international proptech maps, in order to obtain a common framework for future cross-country comparison. Finally, this process allowed us to draw an original definition of proptech.

\section{Results}

\subsection{Schematics and diagrams}

Extant representations of the proptech panorama are elaborated through schematics and diagrams that, from time to time, offer a different interpretive angle. Some of them propose overlapping levels:

(1) One level (e.g. MIPIM - Plate 1). These offer a typological grouping of proptech companies, which aims at describing the extent of technological innovation;

(2) Two levels (e.g. CBInsights - Plate 2). These reflect a differentiation of the activities by asset class, namely residential, commercial or both, with an overlapping level addressing the extent of technological innovation; and

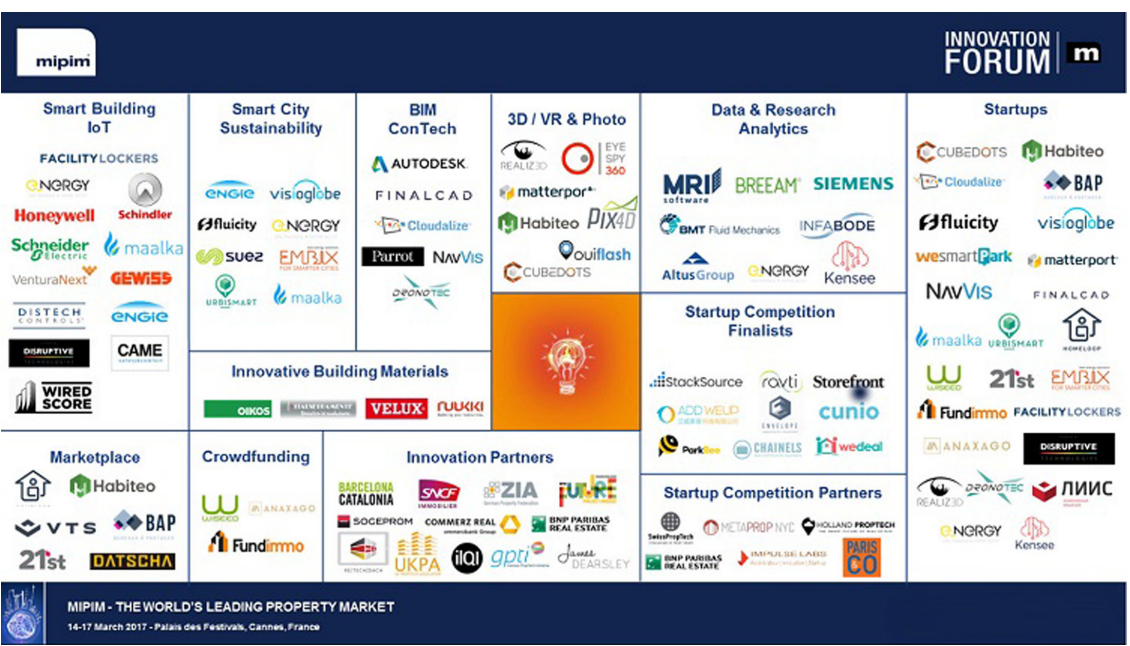

Plate 1.

MIPIM PropTech map

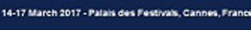




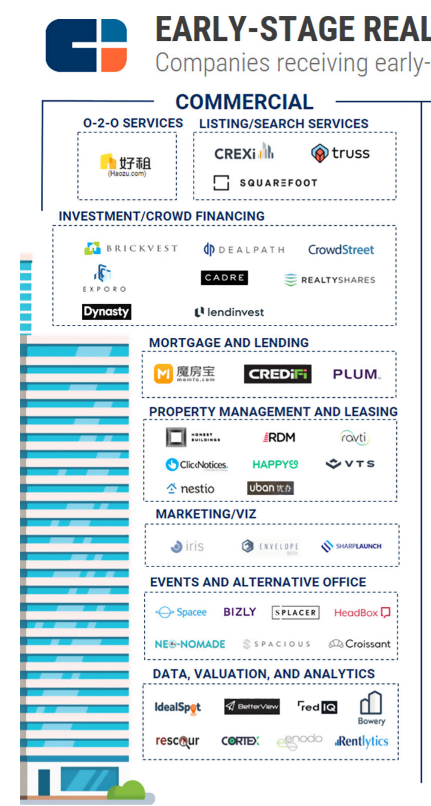

EL ESTATE TECH MARKET MAP
$y$-stage financing (seed or Series A) since 201.5

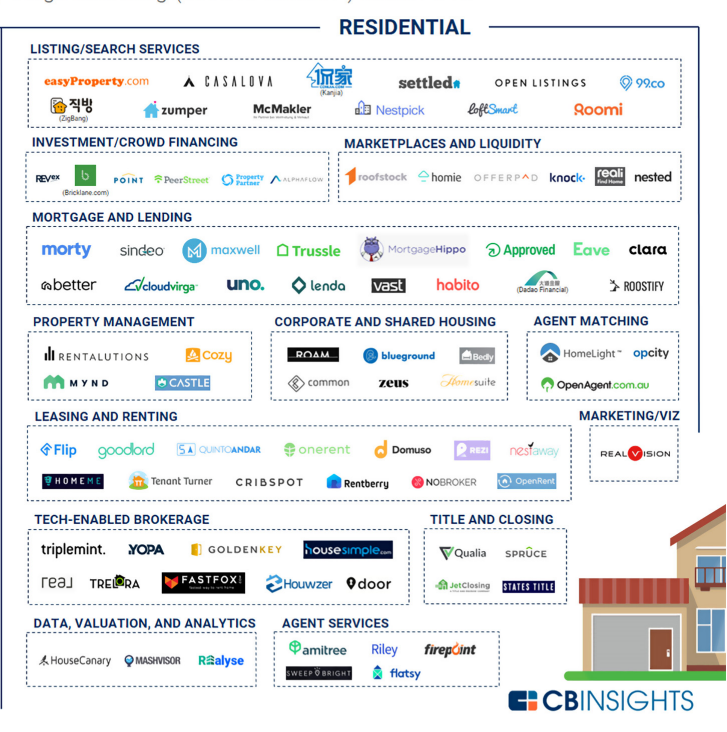

Note(s): Some companies on this graphic have raised follow-on rounds past Series A, including Series B and Series C financings
R.E. property meets technology

131

Plate 2.

CBInsight PropTech map

(3) Matrix of criteria where verticals and horizontals intersect to give a more complex interpretation of the innovation drivers and subsectors (e.g. Baum, 2017-Figure 1).

Maps can be distinguished also by graphical solutions that help visualize different aspects, as follows:

(1) Bubble charts have the advantage of showing the relative dimension of the different classes and make it possible to depict business relations (e.g. James Dearsley's UK map - Plate 3);

(2) Tree diagrams with categories and subcategories allow for a schematic demonstration of the complexity of different business areas (e.g. CBInsights Plate 2);

(3) Tables help represent different sectors and are functional to explain matrixes (e.g. Baum, 2017-Figure 1).

\begin{tabular}{|l|l|l|l|}
\hline \multicolumn{2}{|c|}{ Real Estate FinTech } & Shared Economy & Smart Real Estate \\
\hline Information & yes & yes & yes \\
\hline Transactions/marketplace & yes & yes & \\
\hline Management/control & & & yes \\
\hline
\end{tabular}

Figure 1.

Baum's (2017) PropTech taxonomy 
JPIF
39,2

132

Plate 3.

James Dearsley's UK PropTech map

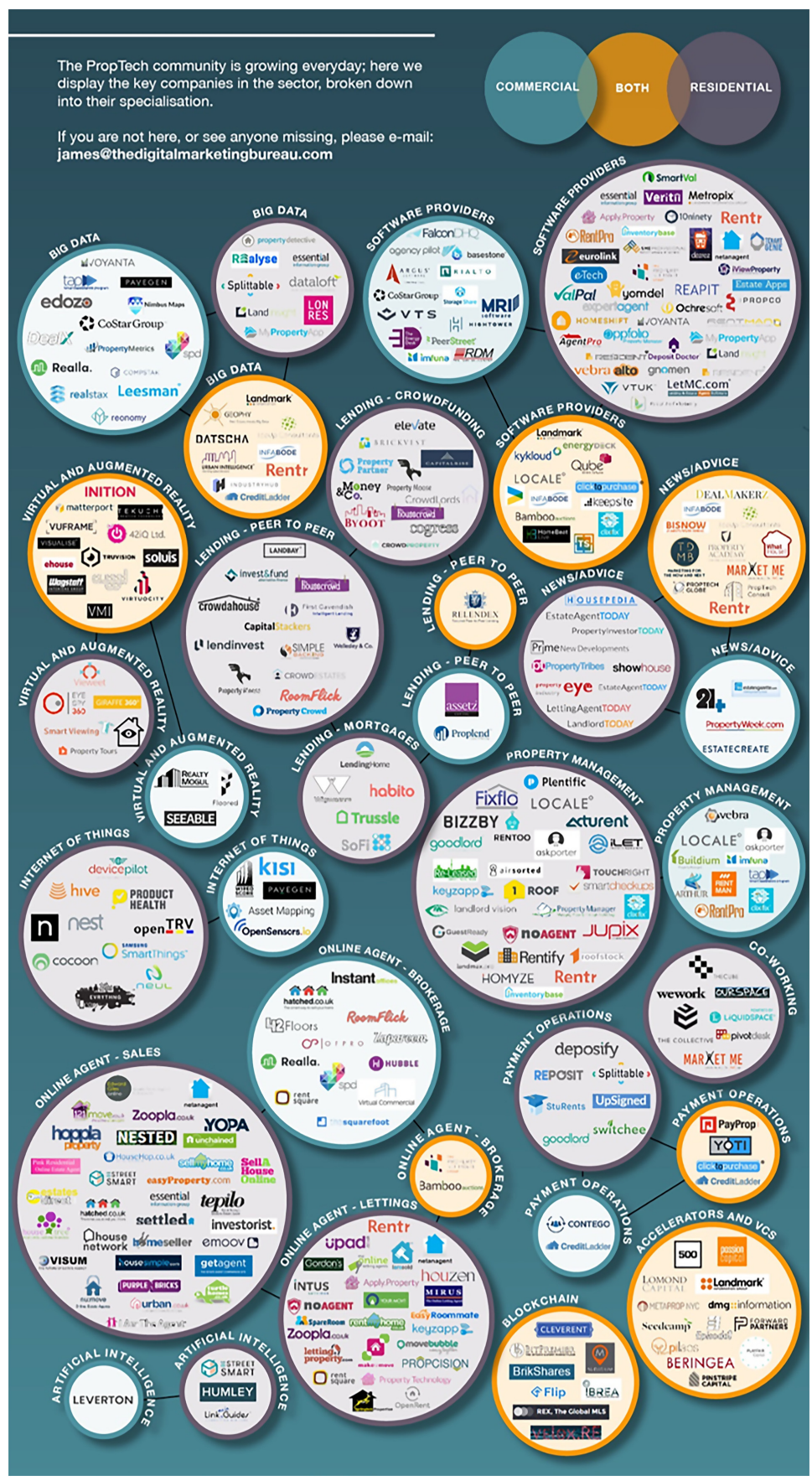


Finally, existing maps are applied at different scales:

(1) Supranational, for example, Venture Scanner (Venture Scanner, 2018b), CBInsights (Yatskevich, 2018), MIPIM (Yatskevich, 2018) and Baum (2017) for a global view and JLL (2017) for the Asia-Pacific area; and

(2) National, for example, the United Kingdom (Dearsley, 2017), Germany (GewerbeQuadrat, 2018), Spain (Spanish Estate, 2018), Finland (Käki, 2018), France (Flattin, 2017).

\subsection{Proptech categories}

A cross-interpretation of the proptech maps representing the industry generated 14 general categories (Table 1). Not all of them exist transversally in both supranational and national maps. Five to ten categories can be found in a single source.

Contech is a disputed category. Baum's (2017) argument is reasonable that contech belongs to the construction world rather than the real estate world. Even if the engineering and construction sector could potentially converge in the real estate world soon, for now they can still be identified as distinct environments, with their own processes and progress. However, eight sources out of ten mention it in the proptech panorama.

The most common categories are: digital space (ten sources); smartness and IoT (ten sources); financial services (ten sources); facility, property and asset management (ten sources); and intermediation/disintermediation (nine sources). The latter three clusters all entail a range of activities that strongly characterize the real estate sectors in the analog domain. Hence, in the proptech world these services are reinterpreted in a digital logic. Digital space and smartness and Io $T$ are probably the first digital applications on buildings and real estate assets (i.e. Proptech 1.0), thus it is not surprising that they are quite common across countries and well-established across sources.

Generally, national categorizations tend to present a range of management services that do not appear in supranational categories. These regard professional services, blockchain, data and analytics, news/reviews and support to digital real estate businesses.

\subsection{Italian companies}

In Italy, 43 proptech-related businesses were collected. Most of the list (33\%) has been collated through general Internet searches (Figure 2). Fintastico and PoliHub give access to only a residual number of proptech companies, respectively 9 and $5 \%$ of the total, while Assolombarda seems the only structured effective database, providing $30 \%$ of the companies' names. Unfortunately, though, this acts at a regional level and does not reflect data at national scale. Snowball sampling suggested $23 \%$ of the companies. This fragmented landscape can have affected analysis, especially for what concerns geographic localization. It must be noted that the lack of a unified and trusted source where one can spot proptech companies makes research in this field troublesome and can discourage not only scholars from enquiry but also investors and potential customers from the Italian market.

The year of foundation shows that 2016 is a turning point for the development of this sector in Italy. That year, one-fourth of the start-ups were created (11 proptech). This explosion is in line with the global phenomenon, but it might have been encouraged by a decree on tax incentives for investment in innovative start-ups (interministerial decree of February 25, 2016) released the same year.

Geographic localization is considered in relation to Google and Fintastico sources, since Assolombarda and PoliHub are limited to the Lombardy region as a catchment area. The highest density of proptech gathers in the north of Italy $(88 \%)$, particularly in Milan. This
R.E. property meets technology 


\section{JPIF
39,2}

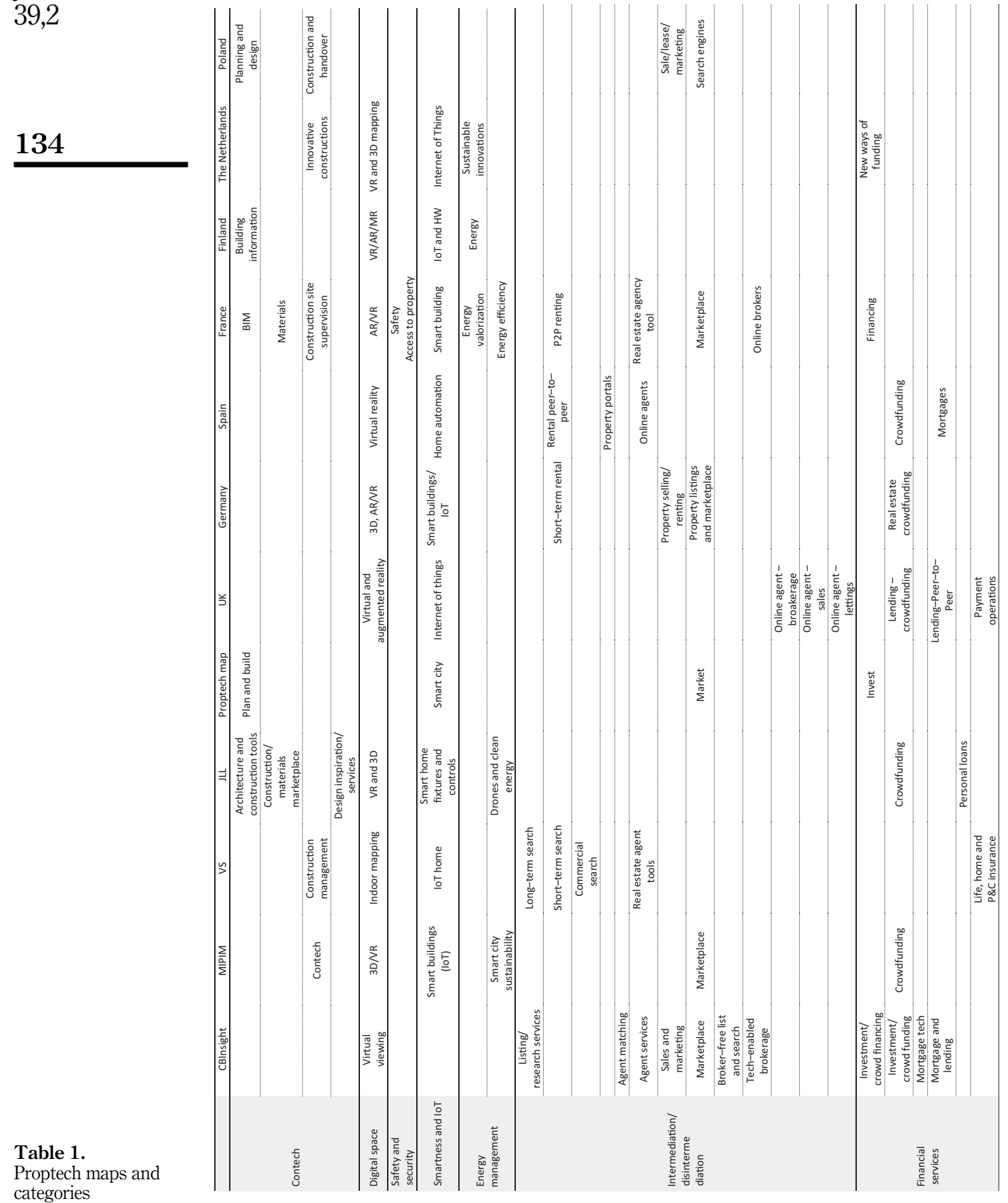




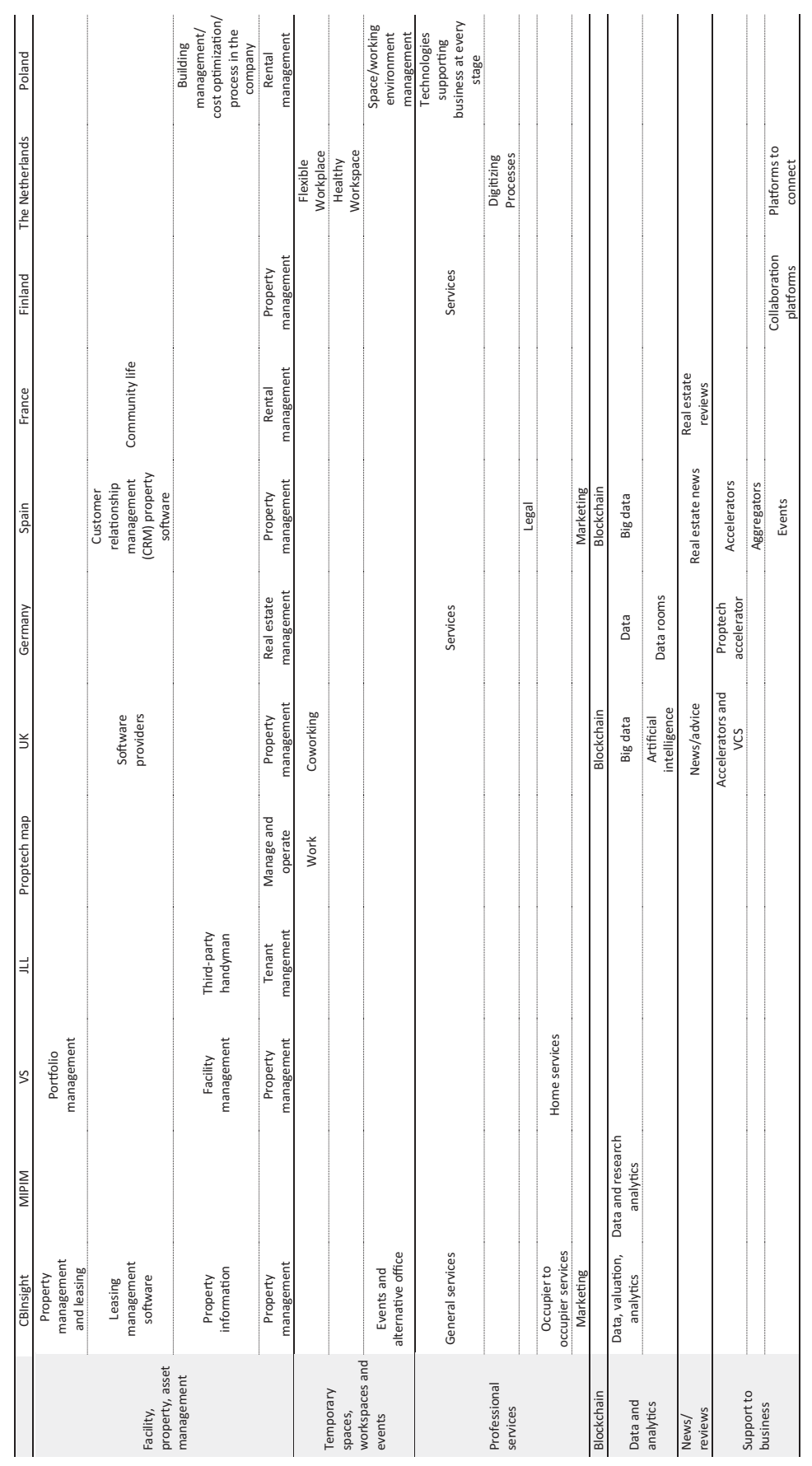

R.E. property meets technology

135

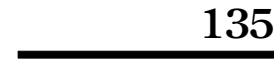


JPIF

39,2

136 phenomenon of concentration has also been registered elsewhere, especially in the London area compared to the rest of the United Kingdom.

The presence of some proptech companies started abroad and operating in Italy emerges, mainly in the Fintastico platform. There are seven, mostly located in the United Kingdom, Spain and Germany, all countries where the number of proptech businesses is very high.

In an international context, 43 companies seem few, especially when compared to other European countries, many of which created hundreds of proptech firms in the last few years. However, the sector has been developing at a fast pace in Italy as well. Unfortunately, the absence of a network and unified search engine entails serious difficulties in measuring the proptech market size on a national scale. Since the beginning of this research, the authors in collaboration with the newly established "Italia Proptech Monitor" and "Proptech Joint Research Center" at Politecnico di Milano have empirically grasped a significant increase in the number of Italian proptechs but have been unable to update and elaborate this information further.

Regarding proptech categories, based on the aforementioned reasoning, contech companies have been excluded from the present scouting in Italy. In all, the 43 Italian proptech companies existing in December 2018 can be ascribed to 13 business segments, as follows:

(1) Virtual reality;

(2) Smart building/IoT;

(3) Short-term rental and hospitality;

(4) Brokerage;

(5) Investment and auction;

(6) Crowdfunding;

(7) Management (marketing, facility and property);

(8) Workspace and coworking;

(9) Event spaces;

(10) General management;

(11) Consulting;

(12) Marketing;

(13) Blockchain.
Figure 2.

Italian proptech businesses by source

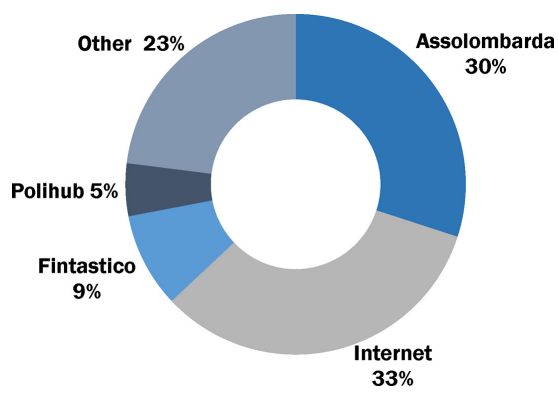


These segments belong to at least eight out of 14 general categories (Table 2). Even though the number of proptech companies in Italy is inferior compared to the other analyzed countries, the existing businesses cover on average a good number of categories, compared with other nations.

It is noticeable that the whole area developing data and analytics, news/reviews and support to digital real estate businesses does not seem to be present in this country.

\subsection{Proptech framework}

Inspired by theoretical approaches aiming at proptech classification, the categories that have been derived through a bottom-up approach can be merged into few overarching classes. To do so, the three verticals proposed in Baum's (2017) model have been slightly refined and integrated (Table 3). "Smart real estate" and "Fintech" are clearly the most developed macrosectors. The first including: digital space, safety and security, smartness and IoT and energy management; and the second composing of: intermediation/disintermediation and financial services. Facility, property and asset management and temporary spaces, workspace and events, could be included in the "Sharing economy" cluster established by Baum (2017). However, they include a range of technologies that not only are supposed to be shared by multiple users but more extensively entail the development of new models of offering goods by providing consumers with services rather than with products. Thus, they can be identified more appropriately as "Product-service systems." One additional class can be introduced as "Information and knowledge management," which includes professional services, blockchain, data and analytics, news/reviews and support to digital real estate businesses.

\section{Discussion}

Schematics and diagrams show a disorganized approach to proptech mapping. It is clear that each organization applies its own conceptual and graphical method to build up clusters. Apart from the natural inhomogeneity of the market across different countries, representations and definitions of market segments vary so much that often it is challenging to compare experiences or figure out differences and similarities. Just think of the multiplicity of nomenclatures that show up in the "Intermediation/Disintermediation" category. Similar activities are named from time to time "Sales and marketing" (CBInsight),

\begin{tabular}{ll}
\hline Proptech categories & Italian business segments \\
\hline Contech & Virtual reality services \\
\hline Digital space & \\
\hline Safety and security & Smart building/loT \\
\hline Smartness and loT & \\
\hline Energy management & Short-term rental and hospitality \\
\hline Intermediation/disintermediation & Brokerage \\
\hline Financial services & Investment and auction \\
\hline Facility, property, asset management & Crowdfunding \\
\hline Temporary spaces, workspace and events & Management (marketing, facility and property) \\
\hline Professional services & Workspace and coworking \\
\hline Blockchain & Event space \\
\hline Data and analytics & Consulting \\
\hline News/reviews & General management \\
\hline Support to digital real estate businesses & Marketing \\
\hline
\end{tabular}

R.E. property meets technology 
JPIF

39,2

138

\begin{tabular}{|c|c|c|c|c|c|c|c|c|c|c|c|c|c|c|c|c|c|c|}
\hline Кјеұl & $m$ & $>$ & - & & $\bullet$ & & $\bullet$ & $\bullet$ & $\bullet$ & & $\bullet$ & & $\bullet$ & $\bullet$ & & & & $\infty$ \\
\hline puejod & กี & $\bullet$ & & & & & $\bullet$ & & $\bullet$ & & $\bullet$ & & $\bullet$ & & & & & in \\
\hline spuе|رәцдәN әપЦ & 각 & $\bullet$ & $\bullet$ & & $\bullet$ & $\bullet$ & & $\bullet$ & & & $\bullet$ & & $\bullet$ & & & & $\bullet$ & $\infty$ \\
\hline pueןu! & 을 & $\bullet$ & • & & $\bullet$ & - & & & - & & $\bullet$ & & & & & & - & $N$ \\
\hline әכueג & คิ & $\bullet$ & $\bullet$ & $\bullet$ & $\bullet$ & $\bullet$ & - & $\bullet$ & $\bullet$ & & & & & & & - & & a \\
\hline u!̣eds & $\infty$ & & - & & $\bullet$ & & $\bullet$ & $\bullet$ & - & & & & $\bullet$ & - & $\bullet$ & $\bullet$ & - & 엄 \\
\hline Киещләэ & 只 & & - & & $\bullet$ & & • & $\bullet$ & • & & & & $\bullet$ & & $\bullet$ & & $\bullet$ & $\infty$ \\
\hline त्रก & 6 & & $\bullet$ & & $\bullet$ & & $\bullet$ & $\bullet$ & $\bullet$ & & $\bullet$ & & $\bullet$ & $\bullet$ & & $\bullet$ & $\bullet$ & 엄 \\
\hline deس પગәдdoıd & in & $\bullet$ & & & $\bullet$ & & $\bullet$ & $\bullet$ & $\bullet$ & & $\bullet$ & & & & & & & $\omega$ \\
\hline 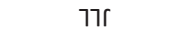 & $\stackrel{\pi}{\forall}$ & - & $\bullet$ & & $\bullet$ & $\bullet$ & & $\bullet$ & $\bullet$ & & & & & & & & & 6 \\
\hline$S \wedge$ & $\stackrel{m}{m}$ & $\bullet$ & $\bullet$ & & $\bullet$ & - & $\bullet$ & $\bullet$ & $\bullet$ & & & & $\bullet$ & & & & & $\infty$ \\
\hline WIdIW & $\stackrel{\pi}{\sim}$ & $\bullet$ & $\bullet$ & & $\bullet$ & $\bullet$ & $\bullet$ & $\bullet$ & & & & & & & $\bullet$ & & & $N$ \\
\hline 74જిเsu|gว & $\stackrel{\pi}{\sim}$ & & $\bullet$ & & & & $\bullet$ & $\bullet$ & $\bullet$ & & $\bullet$ & & $\bullet$ & & $\bullet$ & & & $N$ \\
\hline 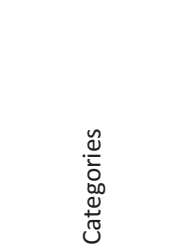 & 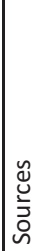 & 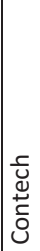 & 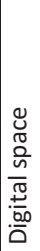 & 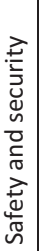 & 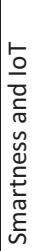 & 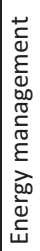 & 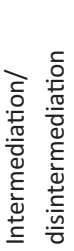 & 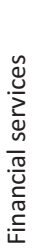 & 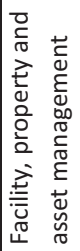 & 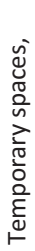 & 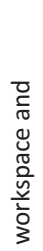 & 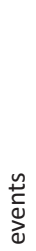 & 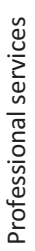 & $\begin{array}{l}. \frac{\subseteq}{0} \\
\frac{c}{U} \\
\frac{v}{U} \\
\frac{0}{D}\end{array}$ & 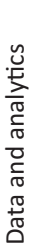 & $\frac{n}{3}$ & 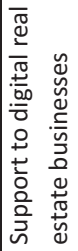 & 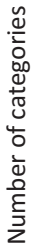 \\
\hline 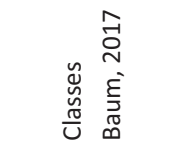 & & 岳 & & 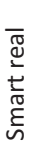 & 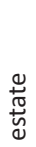 & & 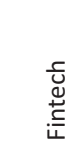 & & s & & & & & & & & & \\
\hline 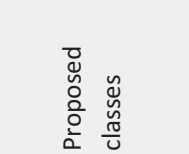 & & 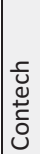 & & $\begin{array}{l}\overline{\mathscr{U}} \\
\frac{1}{L} \\
\frac{t}{0} \\
\frac{E}{n}\end{array}$ & 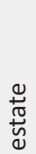 & & 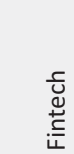 & & $\begin{array}{l}\text { t' } \\
\text { ํํㅁ } \\
\text { 은 }\end{array}$ & $\frac{0}{d}$ & $\frac{n}{\frac{n}{\nu}}$ & & & 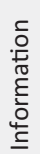 & $\underset{\sigma}{\frac{D}{\sigma}}$ & 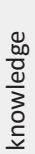 & 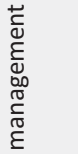 & \\
\hline
\end{tabular}

Comparative scheme of supranational and national

proptech maps 
"Property selling/renting" (Germany) or "Sale/lease/marketing" (Poland) across different sources. Online agency can include up to three distinct subsegments in a single source (United Kingdom), that is, brokerage, sales and lettings. However, at the same time, other sources refer to it as "Agent services" (CBInsight), "Real estate agent tools" (VS), "Online agents" (Spain) and "Real estate agency tool" (France).

It is worth noting that such a variety of wording, while expressing the complexity of the activities that belong to proptech, might create misleading analyses. It would be important for these dispersed proptech maps to converge progressively under some unified categories and definitions. This way cross-country and cross-organization comparisons would become both easier and more reliable. At this point, it would be interesting to experiment with different visualization solutions, rather than choosing only one, as they can return different information depending on the study goal.

The juxtaposition of maps shows that on average supranational maps tend to collect six to eight categories, basically trying to synthetize in a few meaningful sectors multiple proptech activities. However, many of them are missing relevant categories that are present at national scales. For example, "Temporary spaces, workspace and events" is common to the United Kingdom, Finland, the Netherlands, Poland and Italy, but is not mentioned in MIPIM, nor in VS and JLL. National maps, where country-specific characteristics emerge, span from five to ten categories (respectively Poland and the United Kingdom). However, categories that cover one single country, thus defining a peculiar attitude toward specific applications, are also lacking, except for "Safety and security" that appears only in France. A question arises whether supranational maps should more carefully observe national trends, or national networks should try to adapt their maps to those proposed by major global platforms. Moreover, with the goal of letting novel emerging phenomena pop up, it seems necessary to propose a multilevel representation. Categories and subcategories can explain both convergent and divergent market dynamics to discern similarities and differences.

The Italian case, which remained hidden until the present study, seems promising. The active proptech categories are well aligned overall with the other main international experiences, even though the number of proptechs populating them is still small. The Italian proptech sector is in its infancy, but the context is rapidly changing. The lack of activities such as "Data and analytics," "News/reviews" and "Support to digital real estate businesses," which are present in many of the other analyzed countries, might have contributed to the backwardness of Italian proptech. The absence of online databases and websites to collect data and share information on the sector's development reduces knowledge exchange and opportunities for collaboration. The blurred panorama of the Italian proptech sector probably discourages single entrepreneurs from establishing new relationships and meaningful contacts with other innovative businesses. Given the lack of a database collecting proptech companies and the absence of events organized to push cross-pollination, it is not surprising that ventures are slow in developing new business opportunities.

Finally, the newly proposed classes aim at providing an interpretation of the proptech subsectors that also combines the innovation drivers and builds up a broader definition of proptech.

Baum (2017) tried to describe the proptech sector into three verticals of "Smart real estate," "Shared Economy" and "Real Estate Fintech," corresponding to technology-based platforms that facilitate, respectively, (a) operation and management of real estate assets; (b) the use of real estate assets; and (c) the trading of real estate ownership. These verticals have been criticized by Shaw (2018) for being ill-defined, in particular the concept of sharing economy. With a bottom-up approach that develops from categories analysis, here the authors propose a different and broader interpretation of those verticals. To do so, the authors argue that a proptech definition cannot prescind from a technology taxonomy, in
R.E. property meets technology 
JPIF

39,2

140

support of a deeper understanding of the embodied sociotechnical mechanisms (Shaw, 2018). Baum's horizontals defining market processes (information, transactions, control) could be fruitfully substituted with technological innovation drivers. The English Royal Society for the Encouragement of Arts, Manufactures and Commerce (RSA, 2019) builds a framework to make sense of the impact of the current technology on the quantity and quality of work. The framework is broad enough to explain also the impact of technology on real estate processes, involving:

(1) Automation, where technology completes tasks or modifies who is responsible for undertaking them (e.g. self-service checkouts);

(2) Brokerage, where technology mediates between buyers and sellers, even replacing multiple agents with a single platform (e.g. eBay, Uber, etc.);

(3) Digitization, where technology turns physical goods and knowledge into data and services that can be shared at lower cost (e.g. Netflix);

(4) Management, where technology aids the monitoring and organization of people (e.g. scheduling software).

Based on this concept, automation happens to illustrate well what "Smart real estate" proptech does; brokerage actually describes what proptech in the "Fintech" class works for; digitization defines well the idea of "Product-service systems" applied to proptech; and management fits the class of "Information and knowledge management" (Table 4). These definitions, more than offering a description of proptech classes, can help grasp the complexity and variety of the proptech environment.

Accordingly, an overarching definition of proptech is: "technology that interacts with real estate processes by: completing tasks or modifying responsibilities, mediating between buyers and sellers, turning physical goods and knowledge into data and services, and aiding people's organization".

\section{Conclusions and limitations}

The present paper aimed to:

(1) Analyze existing proptech categories;

Table 4.

Interpretive scheme

\begin{tabular}{|c|c|c|c|c|}
\hline & Smart real estate & Fintech & $\begin{array}{c}\text { Product-service } \\
\text { systems }\end{array}$ & $\begin{array}{c}\text { Information and } \\
\text { knowledge } \\
\text { management }\end{array}$ \\
\hline Automation & $\begin{array}{l}\text { Completes tasks or } \\
\text { modifies who is } \\
\text { responsible for } \\
\text { undertaking them }\end{array}$ & & & \\
\hline Brokerage & & $\begin{array}{c}\text { Mediates between } \\
\text { buyers and sellers, } \\
\text { even replacing multiple } \\
\text { agents with a single } \\
\text { platform }\end{array}$ & & \\
\hline Digitization & & & $\begin{array}{c}\text { Turns physical goods } \\
\text { and knowledge into } \\
\text { data and services that } \\
\text { can be shared at low } \\
\text { cost }\end{array}$ & \\
\hline Management & & & & $\begin{array}{c}\text { Aids monitoring and } \\
\text { organization of people }\end{array}$ \\
\hline
\end{tabular}


(2) Advance the current knowledge on Italian proptech; and

(3) Propose a critical definition of proptech.

Through the collection and analysis of several proptech maps, recurring clusters and categories were outlined. An in-depth scouting of the Italian market allowed a comparison to the international context. Elaboration of the match between existing proptech categories and classes permits us to offer a definition of proptech from a new angle.

In conclusion, proptech mapping would benefit from reference to a common categorization

R.E. property meets technology

framework. Work to develop an appropriate proptech taxonomy should be encouraged in the near future in order to not only analyze trends but also anticipate innovation opportunities. The Italian proptech sector presents characteristics that make it interesting in terms of growth potential. However, without the establishment of an entity able to observe and track market trends, to aggregate proptech experiences and to foster networking, new entrepreneurial initiatives will still be slow to develop. Finally, a framework is proposed to define proptech starting from the impact of new technology on the real estate processes and to include the broadest array of innovations in processes, products, services and market.

This research had two main limitations. First, the study elaborates on a relatively small number of proptech maps. Collecting more maps worldwide and comparing them is one of the next goals for expanding the research. Second, the snowball sampling method seems insufficient to gather all the existing proptech companies. Even by combining different sources and databases, grey areas are left. The application of innovative techniques to scout for proptech companies through digital processes (e.g. artificial intelligence) is foreseen to improve the scouting method.

A future object of research could be a focus on the Italian market, where three main topics could be further investigated. First, crowdfunding seems the most promising area. This probably depends on the fact that Italy was the first European country to have introduced a specific and organic regulation for equity crowdfunding in 2012. Crowdfunding is a widely investigated area, counting multiple Scopus-indexed scientific articles. Understanding the specificities of these operations in the Italian proptech market might be worthwhile for supporting entrepreneurship opportunities. Another area of great interest is that of short-term rental and hospitality. Given the large number of nonprimary homes owned by Italian families ("second and third homes"), this area expresses great potential for growth. Proptechs operating in this area are becoming very successful, including those that offer services to support Airbnb (e.g. marketing, concierge, facility and property management) and those that have been developing other innovative building products. Finally, a peculiarity of the Italian market should be noted: various companies in the sector can be traced back to the professional services area (i.e. general management, consulting, marketing). These companies are created with the aim of providing innovative services to professionals and real estate operators, which gives the sense of many traditional real estate enterprises in need of help for transitioning to a digital model. An in-depth analysis of their activities might shed light on novel professional figures that are asked to fill existing voids.

\section{References}

Baum, A. (2017), PropTech 3.O: The Future of Real Estate, University of Oxford, Oxford, Saïd Business School.

CBInsights (2017), "Real estate tech funding reaches new highs in 2016, Research Briefs", available at: https://www.cbinsights.com/research/real-estate-tech-startup-funding/ (accessed 17 October 2018). 
JPIF

39,2

Dearsley, J. (2017), "UK property technology companies: infographic", available at: http://www. jamesdearsley.co.uk/uk-property-technology-companies-ecosystem/ (accessed 7 November 2018).

Dearsley, J. (2018), “What is PropTech?”, available at: http://www.jamesdearsley.co.uk/what-isproptech/ (accessed 7 November 2018).

Flattin, M. (2017), "Mapping of French PROPTECH start-ups - XAngeVC - medium, medium", available at: https://medium.com/xangevc/mapping-of-french-proptech-start-ups-6d7a51eec610 (accessed 10 December 2018).

Gewerbe-Quadrat (2018), “Digital real estate Deutschland August 2018”, Gewerbe-Quadrat”, available at: https://www.gewerbe-quadrat.de/digital-real-estate-deutschland-august-2018/ (accessed 10 December 2018).

Grünewald, S. (2018), "Real estate innovations overview", available at: https:/assets.kpmg.com/ content $/ \mathrm{dam} / \mathrm{kpmg} / \mathrm{nl} / \mathrm{pdf} / 2018 /$ advisory/real-estate-innovations-overview-2018.pdf (accessed 14 December 2018).

Hasenmaile, F. and Rieder, T. (2017), "PropTech: new kids on the block", Economics Alert, pp. 1-7.

Jacob, E.K. (2004), “'Classification and categorization : a difference that makes a difference”, Library Trends, Vol. 52 No. 3, pp. 515-540, doi: 10.1007/s11406-014-9559-1.

JLL (2017), “Clicks and mortar: the growing influence of proptech”, available at: http://www.joneslanglasalle. com.cn/china/en-gb/Research/proptech-clicks-mortar-apac.pdf (accessed 7 November 2018).

Käki, H. (2018), "PropTech scene insight to Finland" available at: https://www.real-estate-innovation. net/forum/wp-content/uploads/sites/9/PropTechFinland.pdf (accessed 10 December 2018).

Maududy, C.F. and Gamal, A. (2019a), "Literature review: technologies and property development", IOP Conference Series: Earth and Environmental Science, Vol. 396 No. 1, doi: 10.1088/1755-1315/ 396/1/012020.

Maududy, C.F. and Gamal, A. (2019b), "Literature review: the impact of property technology (PropTech) in property development", Proceedings of the 33rd International Business Information Management Association Conference, IBIMA 2019: Education Excellence and Innovation Management through Vision 2020, pp. 5370-5376.

Porter, L., Fields, D., Landau-Ward, A., Rogers, D., Sadowski, J., Maalsen, S., Kitchin, R., Dawkins, O., Young, G. and Bates, L.K. (2019), "Planning, land and housing in the digital data revolution/the politics of digital transformations of housing/digital innovations, PropTech and housing - the view from Melbourne/digital housing and renters: disrupting the Australian rental bond system and Tenant Advocacy/Prospects for an Intelligent Planning System/What are the Prospects for a Politically Intelligent Planning System?", Planning Theory and Practice, Vol. 20 No. 4, pp. 575-603. doi: 10.1080/14649357.2019.1651997.

PropTech house (2019), Demystifying PropTech - a Complete Overview of the European PropTech Ecosystem, PropTech house.

Putzier, K. (2016), "CRE tech: a promise unfulfilled, the real deal. New York real estate news", available at: https://therealdeal.com/2016/08/05/cre-tech-a-promise-unfulfilled/ (accessed 8 November 2018).

Pyle, A., Grunewald, D. and Wright, N. (2017), "Bridging the gap. How the real estate sector can engage with PropTech to bring the built and digital environments together", available at: https://assets.kpmg.com/content/dam/kpmg/uk/pdf/2017/11/proptech-bridging-the-gap.pdf (accessed 17 October 2018).

Real Estate Innovation Network, Expo Real and PwC (2018), "PropTechMap", available at: https:// www.proptechmap.com/ (accessed 13 December 2018).

RICS (2018), "PropTech”, RICS Glossay. available at: https:/www.ricsfirms.com/glossary/proptech/ (accessed 8 November 2018).

RSA (2019), The Four Futures of Work. Coping with Uncertainty in an Age of Radical Technologies, Royal Society for the encouragement of Arts, Manufactures and Commerce (RSA) Action and Research Center, London. 
Shaw, J. (2018), "Platform real estate: theory and practice of new urban real estate markets", Urban Geography, pp. 1-28. doi: 10.1080/02723638.2018.1524653.

Singh, A. (2018), "Global real estate tech/PropTech investment Report for H1 2018 | YoStartups", YoStartups, available at: https://yostartups.com/global-real-estate-tech-proptech-investmentreport-for-h1-2018/ (accessed 10 December 2018).

Sipilä, S. and Haataja, T. (2019), "The Finnish proptech cluster, a global testbed for innovation?", KPMG, available at: https://suuntakasvuun.kpmg.fi/2019/08/14/the-finnish-proptech-cluster-aglobal-testbed-for-innovation/ (accessed 26 November 2019).

SkyConcept (2018), "Map of polish startups. technologies for real estates - SkyConcept", SkyConcept, available at: http://www.skyconcept.pl/blog/map-of-polish-startups-technologies-for-real-estates/ (accessed 14 December 2018).

Spanish Estate (2018), "Spain Proptech map", available at: https:/www.spanishestate.com/proptech (accessed 12 December 2018).

Stanley, A. (2018), PropTech 2.0, The EdgeCushman and Wakefield, , pp. 59-61.

Unissu (2019), "Global PropTech analysis: europe”, available at: https://www.unissu.com/proptechresources/proptech-europe (accessed 26 November 2019).

Venture Scanner (2018a), "Mid-year real estate technology exits analysis", available at: https://www. venturescanner.com/blog/tags/proptech/ (accessed 7 November 2018).

Venture Scanner (2018b), "real estate technology startup highlights - Q2 2018”, available at: https:// www.venturescanner.com/blog/2018/real-estate-technology-startup-highlights-q2-2018 (accessed 12 December 2018).

Yatskevich, O. (2018), "What's brewing in real estate tech: property technology trends, codetiburon", available at: https://codetiburon.com/real-estate-tech-industry-landscape/ (accessed 12 December 2018).

\section{Corresponding author}

Chiara Tagliaro can be contacted at: chiara.tagliaro@polimi.it
R.E. property meets technology 